\title{
The majority are not performing home-exercises correctly two weeks after their initial instruction - an assessor-blinded study
}

Mathilde MF Faber, Malene H Andersen, Claus Sevel, Kristian Thorborg, Thomas Bandholm, Michael Rathleff

Introduction: Time-under-tension (TUT) reflects time under load during strength training and is a proxy of the total exercise dose during strength training. The purpose of this study was to inves-tigate if young participants are able to reproduce TUT and exercise form after two weeks of unsupervised exercises. Material and Methods: The study was an assessorblinded intervention study with 29 participants. After an initial instruction, all participants were instructed to perform two weeks of home-based unsuper-vised shoulder abduction exercises three times per week with an elastic exercise band. The participants were instructed in performing an exercise with a predefined TUT (3sec concentric; $2 \mathrm{sec}$ isometric; 3sec eccentric; 2sec break) corresponding to a total of 240 se-conds of TUT during three sets of 10 repetitions. After completing two weeks of unsuper-vised home exercises, they returned for a follow-up assessment of TUT and exercise form while performing the shoulder abduction exercise. A stretch sensor attached to the elastic band was used to measure TUT at baseline and follow-up. A physiotherapist used a pre-defined clinical observation protocol to determine if participants used the correct exercise form. Results: Fourteen of the 29 participants trained with the instructed TUT at follow-up (predefined target: $240 \mathrm{sec} \pm 8 \%$ ). Thirteen of the 29 participants performed the shoulder abduction ex-ercise with a correct exercise form. Seven of the 29 participants trained with the instructed TUT and exercise form at follow-up. Conclusion: The majority of participants did not use the instructed TUT and exercise form at follow-up after two weeks of unsupervised exercises. These findings emphasize the importance of clear and specific home exercise instructions if participants are to follow the given exercise prescription regarding TUT and exercise form as too many or too few exercise stimuli in relation to the initially prescribed amount of exercise most likely will provide a misinterpre-tation of the actual effect of any given specific home exercise intervention. 
2 The Majority are not Performing Home Exercises Correctly Two Weeks after their

3 Initial Instruction - an Assessor-blinded Study

4 Faber $\mathrm{MD}^{1}$, Andersen $\mathrm{MH}^{2}$, Sevel $\mathrm{C}^{3}$, Thorborg K${ }^{4}$, Bandholm $\mathrm{T}^{5}$, Rathleff $\mathrm{MS}^{6,7}$. 5

6 1: Kjellerup Fysioterapi og Træning, Kjellerup, Denmark

7 2: Fysioterapeutisk Specialistteam, Aarhus, Denmark

8 3: VIA University College, Faculty of Health Sciences, Physiotherapy, Aarhus, Denmark

9 4: Sports Orthopedic Research Center - Copenhagen (SORC-C), Arthroscopic Centre

10 Amager, Copenhagen University Hospital, Italiensvej 1, 2300 Copenhagen S

11 5: Physical Medicine \& Rehabilitation Research - Copenhagen (PMR-C), Department of

12 Occupational and Physical Therapy, Department of Orthopedic Surgery, and Clinical

13 Research Center, Hvidovre Hospital, University of Copenhagen, Copenhagen,

14 Denmark.

15 6: Center for Sensory-Motor Interaction (SMI), Department of Health Science and 16 Technology, Faculty of Medicine, Aalborg University, Denmark

17 7: Department of Occupational and Physiotherapy, Aalborg University Hospital, 18 Denmark

21 Corresponding author

22 Michael Skovdal Rathleff

23 Department of Occupational and Physiotherapy

24 Aalborg University Hospital, Denmark

25 Hobrovej 18-22, 9000 Aalborg

26 Email: michaelrathleff@gmail.com

27

28 Word count: 2,677 


\section{Abstract}

\section{Introduction}

33 Time-under-tension (TUT) reflects time under load during strength training and is a

34 proxy of the total exercise dose during strength training. The purpose of this study was

35 to investigate if young participants are able to reproduce TUT and exercise form after

36 two weeks of unsupervised exercises.

\section{Material and Methods}

The study was an assessor-blinded intervention study with 29 participants. After an initial instruction, all participants were instructed to perform two weeks of home-based unsupervised shoulder abduction exercises three times per week with an elastic exercise band. The participants were instructed in performing an exercise with a predefined TUT (3sec concentric; 2sec isometric; 3sec eccentric; 2sec break) corresponding to a total of 240 seconds of TUT during three sets of 10 repetitions. After completing two weeks of unsupervised home exercises, they returned for a follow-up assessment of TUT and exercise form while performing the shoulder abduction exercise. A stretch sensor attached to the elastic band was used to measure TUT at baseline and follow-up. A physiotherapist used a predefined clinical observation protocol to determine if participants used the correct exercise form.

\section{Results}

Fourteen of the 29 participants trained with the instructed TUT at follow-up (predefined target: $240 \mathrm{sec} \pm 8 \%$ ). Thirteen of the 29 participants performed the shoulder abduction exercise with a correct exercise form. Seven of the 29 participants trained with the instructed TUT and exercise form at follow-up.

\section{Conclusion}

The majority of participants did not use the instructed TUT and exercise form at followup after two weeks of unsupervised exercises. These findings emphasize the importance of clear and specific home exercise instructions if participants are to follow the given exercise prescription regarding TUT and exercise form as too many or too few 
62 exercise stimuli in relation to the initially prescribed amount of exercise most likely will 63 provide a misinterpretation of the actual effect of any given specific home exercise 64 intervention.

\section{INTRODUCTION}

Elastic exercise bands are often used during rehabilitation of patients (Alvarez et al. 2006; Andersen et al. 2010; Andersen et al. 2011; Jensen et al. 2014; Rathleff et al. 2014a; Thomas et al. 2005). An elastic exercise band is a versatile training tool allowing patients to perform different types of home-based training including strength training and injury prevention training (Alvarez et al. 2006; Andersen et al. 2011; Jensen et al. 2014). Using elastic exercise bands during rehabilitation has multiple advantages; they are user-friendly, can be adjusted to different resistances, they are cheap and they do not take up much space (Colado et al. 2010; Matheson et al. 2001; Melchiorri, Rainoldi 2011). Multiple studies have compared the effect of training with elastic exercise bands with the effect of training with free weights (e.g., dumbbells) on neuromuscular activation during exercise. These studies generally show that training with elastic exercise bands activates the contracting agonists to the same level as dumbbells, and therefore, the elastic exercise bands are expected to be just as efficacious in improving strength and reducing pain during a rehabilitation program (Andersen et al. 2010; Beers et al. 2008; Colado et al. 2010; Melchiorri, Rainoldi 2011).

In clinical practice, elastic band exercises are often used during home-based unsupervised training where the physiotherapist provides the patient with an initial instruction on how to perform the exercise. Included in these instructions are load, time under tension (TUT), range of motion (ROM), number of repetitions, sets, pauses between exercises, and the appropriate start position (American College of Sports Medicine 2009; Pereira, Gomes 2003). All these factors are important as they are closely linked to the total exercise dose and thus important for the clinical stimulus received by the patient. Especially TUT seems to play a large role in relation to the total exercise dose received during a training session (American College of Sports Medicine 
92 2009; Andersen et al. 2011; Buitrago et al. 2012; Pereira, Gomes 2003; Tran, Docherty

93 \& Behm 2006). Total TUT refers to the total time of all concentric, quasi-isometric and 94 eccentric contraction-phases in a single training set (Skovdal Rathleff, Thorborg \& 95 Bandholm 2013; Tran, Docherty \& Behm 2006,). In combination with load and 96 movement velocity it is an important strength training descriptor as it reflects the time 97 factor of the strength training stimulus (Buitrago et al. 2012; Gentil, Oliveira \& Bottaro 98 2006; Tran, Docherty \& Behm 2006). Physiologically, a higher amount of TUT has been 99 shown to increase myofibrillar protein synthesis more than a lower amount of TUT after 100 a single, work-matched, strength training session in healthy subjects (Burd et al. 2012;

101 Tran, Docherty 2006). Hence, the quantification of the total TUT of performed strength 102 training of the shoulder abductors is important in order to determine if the executed 103 training constitutes a sufficient clinical and physiological stimulus (Buitrago et al. 2012;

104 Burd et al. 2012; Gentil, Oliveira \& Bottaro 2006; Goto et al. 2009; Munn et al. 2005; 105 Nogueira et al. 2009; Toigo, Boutellier 2006; Tran, Docherty 2006; Tran, Docherty \& Behm 2006).

After a single initial instruction, the patients often complete their exercises unsupervised at home and return for follow-up assessment after, e.g., two weeks (Andersen et al. 2011; Beers et al. 2008; Rathleff et al. 2014a; Thomas et al. 2005). During the execution of the home exercises is it assumed that the patient follows the exercises as instructed and prescribed by the physiotherapist and thereby receives the exercise dose

113 intended by the physiotherapist. However, the question remains if the patients are able

114 to follow the exercise prescription and perform the exercise as instructed after only a 115 single initial instruction as common in clinical practice. Clinical experience suggests that exercises are performed too fast at follow-ups thus reducing TUT. A correct execution of

117 home-based exercises is vital in order to know the exercise dose received by the 118 patients during their home-based unsupervised exercises. Exercise dosages that are 119 either too small or too large in relation to the initially prescribed dosage introduce 120 misinterpretation when concluding on effect estimates of any specific intervention in 121 both clinical scenarios and in research. 
123 Therefore, the purpose of this study was to investigate if young participants were able to 124 reproduce TUT and exercise form after two weeks of unsupervised shoulder abduction. 125 This exercise was selected as it has previously shown to be effective in reducing pain in 126 the neck and shoulder muscles (Andersen et al. 2011; Andersen et al. 2008b; Andersen 127 et al. 2008a; Salo et al. 2010; Walther et al. 2004). The main hypothesis was that 128 participants would perform a shorter TUT at follow-up and thus not perform the exercise 129 as instructed. 


\section{METHODS}

\section{Design}

133 This study was designed as an assessor-blinded intervention study with baseline 134 measurements and follow-up after 14 days of unsupervised home-based exercises.

\section{Ethical Considerations}

137 According to the Helsinki-declaration, all participants were provided with verbal and

138 written information about the purpose of the study (WMA Declaration of Helsinki 2013).

139 They signed a written consent as part of the duty to the Ethics Committee

140 (Justitsministeriet 2000), and The Central Denmark Region Committee on Health

141 Research Ethics approved the study (ref: 80/2014).

\section{Setting and Participants}

144 The study was conducted at VIA University College in Aarhus, Denmark, and at a 145 hospital in Denmark. Young pain-free physiotherapy students from VIA University 146 College, Aarhus, were recruited through advertisements at VIA University College, 147 Aarhus. The participants were informed that the purpose of the study was to investigate 148 whether physiotherapy students were capable of reproducing a shoulder exercise 14 149 days after their initial exercise instruction. The information to the participants was kept 150 short in order to blind them for our primary outcome (TUT). Inclusion criteria included 151 fully active range of motion (AROM) in the shoulder joint and no pain in the shoulder 152 region

\section{Equipment}

156 A stretch sensor was used to measure TUT at baseline and follow-up but not during the 15714 days of unsupervised home-based exercise. The stretch sensor was based on the 158 technology designed by Danfoss PolyPower. It acted as an elastic capacitive material 159 stretchable in one direction. It allowed us to measure how much the sensor was being 160 stretched (for further details refer to Kappel et al (Kappel et al. 2012)). The sensor was 
161 attached to the rubber band through two clips that made the sensor easily transferable

162 to other elastic exercise bands. The sensor was attached via a USB to a small box that

163 recorded data 200 times per second. A switch was mounted in the handle of the elastic

164 exercise band so that the data recording would start whenever the handle was pressed.

165 The participants were told that the sensor could measure if the exercise was performed

166 correctly. They were not informed about the primary purpose of measuring TUT.

168 The elastic bands were standard red and green Thera-Bands, which are commonly 169 used in rehabilitation studies (Andersen et al. 2008a; Andersen et al. 2008b; Andersen

170 et al. 2011; Salo et al. 2010; Walther et al. 2004). To ensure that each strength exercise

171 scenario was performed with the same external resistance, corresponding to a relative

172 load of 12 repetition maximum (RM), lines were drawn for every $5 \mathrm{~cm}$ in the entire

173 length of the exercise band. The $12 \mathrm{RM}$ load was determined prior to data collection

174 and reflected a load typically prescribed to treat conditions of the shoulder and neck

175 (Andersen et al. 2008a; Andersen et al. 2008b; Andersen et al. 201; Salo et al. 2010;

176 Walther et al. 2004).

177

178

179

180

181

182

183

184

185

186

187

188

189

190

\section{Test Procedure}

The participants were instructed to perform a shoulder abduction exercise using a Thera-Band. This specific exercise has proven effective in reducing pain in the neck and shoulder muscles (Andersen et al. 2008a; Andersen et al. 2008b; Andersen et al. 2011, Salo et al. 2010, Walther et al. 2004), and this type of live instruction has previously shown to be effective in learning exercises and reflects clinical practice (Reo, Mercer 2004). The instruction was to stand with hip width distance between the feet and place one end of the elastic band under the foot and the other in the hand. Then the subject was to raise the arm slightly in front of the body from 0-90 degrees shoulder abduction and 30 degrees horizontal shoulder flexion. The elbow was to be in a slightly flexed position during the entire range of motion. The palm was to face the floor. The participants were instructed that tension was to be present in the elastic band at 0 degrees' abduction (see figure 1). 
192 Figure 1 here.

194 Further, they were instructed that each exercise session should consist of three sets of 19510 repetitions at 12 RM with a two-minute break between each set. A mark was made 196 on the elastic band at a length corresponding to 12 RM. TUT was 3 sec concentric

197 (con); 2 sec isometric at 90 degrees' abduction; 3 sec eccentric (ecc); 2 sec pause at 0 198 degrees' abduction. Therefore, total TUT during the three sets of 10 repetitions was 240 199 sec.

201 During the initial instruction, the participants received clear and thorough instructions on how to perform the shoulder abduction exercise using the prescribed TUT and the correct exercise form. First, the participants saw the assessor perform the exercise correctly, and afterwards the participants performed the exercise themselves with feedback from a metronome to guide the correct TUT. The participants were given up to five attempts to find the correct TUT by following the beat of the metronome. After the practice, they had a two-minute break before the data collection. Each participant then completed 3 sets of 10 repetitions, with a two-minute beak between the sets. The participants were verbally guided through the test if they did not follow the beat of the metronome, or if they did not use correct exercise form. Therefore, all participants performed the exercise correctly at baseline. After the exercise, the participants were

212 given a training diary as well as date and time for the follow-up. They were informed that

213 the exercise had to be performed at home three times every week for two weeks. The 214 exercise instruction lasted 7-10 minutes and resembled the time spent on exercise 215 instructions for one exercise in normal clinical practice.

217 Fourteen days later the participants returned for the follow-up assessment and were 218 asked to perform three sets of 10 repetitions, using the exercise form as instructed at 219 the first visit and practised at home for the past two weeks. At the follow-up assessment no verbal feedback was given and the metronome was not turned on. The participants were also asked to hand over their training diary to the assessor. 


\section{Outcomes}

The primary outcome was defined as the total TUT during all three exercise sets with the aim to investigate if the participants were capable of reproducing total TUT during the shoulder abduction exercise. Matlab 2011a (the mathWorks, Nattick, USA) was used to analyse the data from the stretch-sensor. A custom-written Matlab programme transformed the stretch sensor data into an image showing the stretch of the sensor as a function of time. The rating of every repetition was done manually by moving the mouse cursor to the visually observed time points corresponding to the contractionspecific phases. This approach has previously been used and demonstrates good validity and reliability (Skovdal Rathleff, Thorborg \& Bandholm 2013).

The secondary outcome was the exercise form. This was measured using a clinical observation form similar to a previous study (Reo, Mercer 2004). The following parameters were noted: hip width distance between the feet, 0-90 degree shoulder abduction, 30 degree horizontal flexion, palm facing the floor, slight flexion of the elbow, tension in the elastic exercise band at 0 degrees' shoulder abduction and alignment though the standing position. The assessor noted the number of errors in the exercise execution by visual observation.

\section{Rating of Data}

The assessor was a female physiotherapist with no previous experience in rating data from the stretch sensor. The assessor received one hour of practice rating data from the stretch sensor using data from participants who were not part of the current study. The rating of data was carried out after completion of all measurements from baseline to follow-up. After this the collected data from each participant, from both baseline and follow-up, were anonymised and given a random number. The assessor was blinded and therefore, did not know whether the data were from baseline or follow-up. Further, the assessor did not know which of the three exercise sets she was rating. 


\section{Intertester Reliability}

254 Before commencing the current study, a pilot study was performed where two 255 independent participants performed six sets of 10 repetitions. Two independent 256 assessors rated the stretch sensor data. This analysis was performed to determine the 257 interrater reliability and an estimate of the worst-case scenario of reliability (it was 258 expected that intrarater reliability would be higher). The intraclass correlation coefficient 259 (ICC 2.1) was used as a relative expression of the reliability while limits of agreement 260 (LoA) were used as an absolute expression of agreement. The ICC for the interrater 261 reliability for single TUT was 0.93 . The agreement between raters for TUT ranged from 2620.57 to 0.56 seconds ( $8 \%$ of mean TUT). Therefore, the predefined target for total TUT was 240 seconds $\pm 8 \%$ (limits: $220.8-259.2$ seconds) to reflect measurement uncertainty.

\section{Sample Size}

As the main hypothesis was that the participants would use a shorter TUT at follow-up, the study was powered to detect a 20 -second lower TUT (approximately $8 \%$ of the mean TUT) at follow-up compared with baseline. Using a standard deviation of 25 seconds at $5 \%$ significance and $80 \%$ power, it was necessary to include 25 participants. To account for potential dropouts, we included 32 participants.

271

272 Statistical Analysis

273 All calculations were performed using Stata version 11 (StataCorp, College Station, 274 Texas, USA). Descriptive statistics are presented as mean and standard deviation. The

275 level of statistical significance was set as $P<0.05$. Parametric statistics were used 276 because the data were normally distributed. Total TUT from baseline to follow-up was 277 compared using paired t-tests. 


\section{RESULTS}

281 A total of 35 participants signed up for the study, but three of them had shoulder pain and did not fulfil the inclusion criteria and were excluded from the study. A total of 32 physiotherapy students aged 20-27 (10 men and 19 women) fulfilled the inclusion criteria and were included. Further three participants dropped out during the two weeks of unsupervised exercises: Two sustained an injury to the arm (unrelated to the exercise) and could not participate, and one did not turn up for follow-up for unknown reasons. Therefore, 29 participants completed both baseline and follow-up visits.

Twenty of the 29 participants completed the six exercise sessions over two weeks as prescribed. One participant completed the exercise more than the recommended 6 times, and 8 completed the exercise less than recommended. One participant completed the exercise three times, two participants completed the exercise four times and five participants completed the exercise five times during the 14 days.

Total TUT at baseline was $255.2 \mathrm{~s}( \pm 10.6)$. At follow-up, total TUT was $252.5 \mathrm{~s}( \pm 41.0$ s.) with a range from 160 seconds and up to 370 seconds. No significant difference was detected in total TUT between baseline and follow-up (2.6 s 95\% Cl: -13.2 ; 18.5) p=0.74). Fourteen of the 29 participants used the instructed TUT at follow-up (predefined target: $240 \mathrm{~s} \pm 8 \%$ ). Six used a lower TUT and 15 used a higher TUT than instructed (Figure 2).

Figure 2 here.

Thirteen of the 29 participants performed the shoulder abduction with the correct exercise form. Seven of the 29 used both the instructed TUT and exercise form at follow-up. The most common mistakes were; no flexed position in the elbow and abducting the arm to more than 90 degrees (Table 1). 
311

Peer) reviewing PDF | (2015:03:4356:1:0:NEW 29 May 2015) 


\section{DISCUSSION}

314 Contrary to our hypothesis, the participants did not systematically use a lower TUT at

315 follow-up but varied considerably. Half of the participants used the instructed TUT and

316 less than half used the instructed exercise form at follow-up. Physiotherapists may think

317 they know the exercise dose received by the patients during their home-based

318 exercises, but these results suggest that some receive a higher exercise dose, while

319 others receive an exercise dose too low to constitute a sufficient clinical stimulus.

320

321 Practical Relevance of the Study

322 At baseline the participants had a mean total TUT of 255.2 seconds with a standard

323 deviation of 10.6 seconds. At follow-up no significant change appeared in mean TUT,

324 but the standard deviation increased four-fold to 41.0 seconds with a range from 160

325 seconds and up to 370 seconds. If TUT at follow-up reflected the exercise dose during

326 unsupervised exercises, then some participants would receive a 50\% higher exercise

327 dose than instructed, while others would receive a $40 \%$ lower dose. The largest concern

328 would most likely be those receiving a lower exercise dose than intended as it may not

329 elicit a sufficient clinical stimulus. Participants receiving an exercise dose higher than

330 prescribed may end up with a secondary overuse injury hampering the rehabilitation of

331 their original injury. However, the main concern with patients not receiving the

332 prescribed exercise dosage is that decisions on further progression or cessation of a

333 specific program are difficult. It leaves the treating therapist or researcher in doubt

334 whether the lack of progression or the increase in symptoms is due to not following the

335 exercise intervention as prescribed, or whether this is caused by other factors than the

336 actual intervention.

337

338 The large difference in exercise dose may result in different clinical outcomes (Osteras,

339 Torstensen 2010; Rathleff et al. 2014b). Previous studies suggest that the length of TUT

340 influences the physiological response, and longer TUT is associated with larger

341 physiological response (Burd et al. 2012; Munn et al. 2005; Tran, Docherty 2006). The

342 optimal TUT may depend on the goal of the exercise and the response of the patients

343 (American College of Sports Medicine 2009; Carpinelli, Otto \& Winnett 2004). 
344 Nevertheless, TUT needs to be defined when giving exercise prescriptions in order to 345 describe the exercise dose (Fukumoto et al. 2014; McBride et al. 2009; Toigo, Boutellier

346 2006; Tran, Docherty 2006).

348 At baseline the participants received a live instruction of the exercise by a 349 physiotherapist. This approach has previously been shown to improve the performance 350 of an exercise compared to a written leaflet, both immediately after the instruction and 351 after one day (Reo, Mercer 2004). Both the objective and subjective outcome of this 352 study demonstrate that it is difficult for participants to perform the exercise as instructed. 353 These findings support older studies using subjective outcomes to determine if the 354 exercise was performed as instructed and highlight how difficult it can be to perform an 355 exercise correctly after a short initial instruction (Reo, Mercer 2004, Henry, Rosemond 356 \& Eckert 1999). This raises the question of how thorough an initial instruction is to be 357 before the physiotherapist can send off the patient to perform home-based exercises. 358 One way to ensure proper exercise execution would be to perform the exercise under 359 supervision during the first couple of weeks and then slowly transit to home-based 360 exercises. Based on these results, it is assumed that some kind of feedback may be 361 needed to avoid a too low exercise dose. This could be done using a simple metronome 362 on the mobile phone of the patient or using exercise-integrated tools such as the 363 Bandcizer to continuously monitor TUT and adherence to exercise (Rathleff et al. 364 2014c). Indeed, such information collected during every home-based exercise session 365 would help qualify the follow-up visits where a patient is not responding as expected 366 based on the treatment plan.

\section{Strengths and Weaknesses of the Study}

370 The primary outcome, TUT, has previously been shown to be valid and highly reliable 371 and the participants were blinded to the primary outcome (Skovdal Rathleff, Thorborg \& 372 Bandholm 2013). The assessor who rated data was blinded towards the rating of 373 baseline or follow-up data. The assessor judging the exercise form was not blinded, 374 which may have biased the secondary analysis on the exercise form. No TUT data were 
375 measured during the home-based exercises, and it is unknown if the subjects 376 performed their exercise differently while at home. The participants were all 377 physiotherapy students and aware that they were being watched while performing the 378 exercise. Further, it might be speculated that physiotherapy students are better at 379 understanding exercise instructions and thereby have a less steep learning curve 380 compared with, e.g., patients. Therefore, these results are most likely a conservative 381 estimate and it would be expected that an even lower proportion of the patients in 382 clinical practice will perform the exercises using the prescribed TUT and exercise form.

383 It is also possible that physiotherapy students would have less motivation to perform the 384 exercises as instructed than patients because patients suffer from pain and functional 385 limitation which could motivate them to do the exercises as instructed.

\section{Future Research}

389 Future studies should investigate if the current findings also apply to patients with shoulder disorders. There is a need to investigate the amount and type of instruction required in order for the participants to perform the exercise as instructed or if real-time feedback during exercises is required. To further elucidate why the participants are not able to reproduce the exercise, an interview could be performed asking the participants questions on this issue. This could provide valuable information on participant preferences for type and form of the exercise instruction.

\section{Conclusion}

399 Contrary to our hypothesis, the participants did not systematically perform a lower TUT 400 at follow-up but varied considerably, both above and below the instructed TUT. Less 401 than $25 \%$ of the participants performed the instructed TUT and the correct exercise form 402 at follow-up after two weeks of unsupervised home-based exercises. These findings 403 emphasize the importance of clear and specific home exercise instruction if participants 404 are to follow the given exercise prescription regarding TUT and exercise form as too 405 many or too few exercise stimuli in relation to the amount of exercise initially prescribed 
406 most likely will provide a misinterpretation of the actual effect of any given specific 407 home-based exercise intervention. 


\section{References}

409

410

411

412

413

414

415

416

417

Alvarez, R.G., Marini, A., Schmitt, C. \& Saltzman, C.L. 2006, "Stage I and II posterior tibial tendon dysfunction treated by a structured nonoperative management protocol: an orthosis and exercise program", Foot \& ankle international./ American Orthopaedic Foot and Ankle Society [and] Swiss Foot and Ankle Society, vol. 27, no. 1, pp. 2-8.

American College of Sports Medicine 2009, "American College of Sports Medicine position stand. Progression models in resistance training for healthy adults", Medicine and science in sports and exercise, vol. 41, no. 3, pp. 687-708.

Andersen, L.L., Andersen, C.H., Mortensen, O.S., Poulsen, O.M., Bjornlund, I.B. \& Zebis, M.K. 2010, "Muscle activation and perceived loading during rehabilitation exercises: comparison of dumbbells and elastic resistance", Physical Therapy, vol. 90 , no. 4 , pp. 538-549.

Andersen, L.L., Kjaer, M., Andersen, C.H., Hansen, P.B., Zebis, M.K., Hansen, K. \& Sjogaard, G. 2008a, "Muscle activation during selected strength exercises in women with chronic neck muscle pain", Physical Therapy, vol. 88, no. 6, pp. 703711.

Andersen, L.L., Kjaer, M., Sogaard, K., Hansen, L., Kryger, A.I. \& Sjogaard, G. 2008b, "Effect of two contrasting types of physical exercise on chronic neck muscle pain", Arthritis and Rheumatism, vol. 59, no. 1, pp. 84-91.

Andersen, L.L., Saervoll, C.A., Mortensen, O.S., Poulsen, O.M., Hannerz, H. \& Zebis, M.K. 2011, "Effectiveness of small daily amounts of progressive resistance training for frequent neck/shoulder pain: randomised controlled trial", Pain, vol. 152, no. 2, pp. 440-446.

Beers, A., Ryan, M., Kasubuchi, Z., Fraser, S. \& Taunton, J.E. 2008, "Effects of Multimodal Physiotherapy, Including Hip Abductor Strengthening, in Patients with Iliotibial Band Friction Syndrome", Physiotherapy Canada.Physiotherapie Canada, vol. 60 , no. 2, pp. 180-188.

Buitrago, S., Wirtz, N., Yue, Z., Kleinoder, H. \& Mester, J. 2012, "Effects of load and training modes on physiological and metabolic responses in resistance exercise", European journal of applied physiology, vol. 112, no. 7, pp. 2739-2748.

Burd, N.A., Andrews, R.J., West, D.W., Little, J.P., Cochran, A.J., Hector, A.J., Cashaback, J.G., Gibala, M.J., Potvin, J.R., Baker, S.K. \& Phillips, S.M. 2012, "Muscle time under tension during resistance exercise stimulates differential muscle protein sub-fractional synthetic responses in men", The Journal of physiology, vol. 590, no. Pt 2, pp. 351-362. 
444

Carpinelli, R.N., Otto, R.M. \& Winnett, R.A. 2004, "A critical analysis of the ACSM position stand on resistance training: Insufficient evidence to support recommened training protocols", Journal of Exercise Physiology Online, vol. 7, no. 3, pp. 1-60.

Colado, J.C., Garcia-Masso, X., Pellicer, M., Alakhdar, Y., Benavent, J. \& Cabeza-Ruiz, R. 2010, "A comparison of elastic tubing and isotonic resistance exercises", International Journal of Sports Medicine, vol. 31, no. 11, pp. 810-817.

Fukumoto, Y., Tateuchi, H., Ikezoe, T., Tsukagoshi, R., Akiyama, H., So, K., Kuroda, Y. \& Ichihashi, N. 2014, "Effects of high-velocity resistance training on muscle function, muscle properties, and physical performance in individuals with hip osteoarthritis: a randomized controlled trial", Clinical rehabilitation, vol. 28, no. 1, pp. 48-58.

Gentil, P., Oliveira, E. \& Bottaro, M. 2006, "Time under tension and blood lactate response during four different resistance training methods", Journal of physiological anthropology, vol. 25, no. 5, pp. 339-344.

Goto, K., Ishii, N., Kizuka, T., Kraemer, R.R., Honda, Y. \& Takamatsu, K. 2009, "Hormonal and metabolic responses to slow movement resistance exercise with different durations of concentric and eccentric actions", European journal of applied physiology, vol. 106, no. 5, pp. 731-739.

Henry, K.D., Rosemond, C. \& Eckert, L.B. 1999, "Effect of number of home exercises on compliance and performance in adults over 65 years of age", Physical Therapy, vol. 79 , no. 3 , pp. $270-277$.

Jensen, J., Holmich, P., Bandholm, T., Zebis, M.K., Andersen, L.L. \& Thorborg, K. 2014, "Eccentric strengthening effect of hip-adductor training with elastic bands in soccer players: a randomised controlled trial", British journal of sports medicine, vol. 48, no. 4, pp. 332-338.

Justitsministeriet 2000, , Lov om behandling af personoplysninger. Available: https://www.retsinformation.dk/Forms/R0710.aspx?id=828 [2014, June 4].

Kappel, S.L., Rathleff, M.S., Hermann, D., Simonsen, O., Karstoft, H. \& Ahrendt, P. 2012, "A novel method for measuring in-shoe navicular drop during gait", Sensors (Basel, Switzerland), vol. 12, no. 9, pp. 11697-11711.

Matheson, J.W., Kernozek, T.W., Fater, D.C. \& Davies, G.J. 2001, "Electromyographic activity and applied load during seated quadriceps exercises", Medicine and science in sports and exercise, vol. 33, no. 10, pp. 1713-1725.

McBride, J.M., McCaulley, G.O., Cormie, P., Nuzzo, J.L., Cavill, M.J. \& Triplett, N.T. 2009, "Comparison of methods to quantify volume during resistance exercise", 
481

482

483

484

485

486

487

488

489

490

491

492

493

494

495

496

497

498

499

500

501

502

503

504

505

506

507

508

509

510

511

512

513

Journal of strength and conditioning research / National Strength \& Conditioning Association, vol. 23, no. 1, pp. 106-110.

Melchiorri, G. \& Rainoldi, A. 2011, "Muscle fatigue induced by two different resistances: Elastic tubing versus weight machines", Journal of electromyography and kinesiology : official journal of the International Society of Electrophysiological Kinesiology, vol. 21, no. 6, pp. 954-959.

Munn, J., Herbert, R.D., Hancock, M.J. \& Gandevia, S.C. 2005, "Resistance training for strength: effect of number of sets and contraction speed", Medicine and science in sports and exercise, vol. 37, no. 9, pp. 1622-1626.

Nogueira, W., Gentil, P., Mello, S.N., Oliveira, R.J., Bezerra, A.J. \& Bottaro, M. 2009, "Effects of power training on muscle thickness of older men", International Journal of Sports Medicine, vol. 30, no. 3, pp. 200-204.

Osteras, H. \& Torstensen, T.A. 2010, "The dose-response effect of medical exercise therapy on impairment in patients with unilateral longstanding subacromial pain", The open orthopaedics journal, vol. 4, pp. 1-6.

Pereira, M.I. \& Gomes, P.S. 2003, "Movement velocity in resistance training", Sports medicine (Auckland, N.Z.), vol. 33, no. 6, pp. 427-438.

Rathleff, M.S., Bandholm, T., Ahrendt, P., Olesen, J.L. \& Thorborg, K. 2014a, "Novel stretch-sensor technology allows quantification of adherence and quality of homeexercises: a validation study", British journal of sports medicine, vol. 48, no. 8, pp. 724-728.

Rathleff, M.S., Roos, E.M., Olesen, J.L. \& Rasmussen, S. 2014b, "Exercise during school hours when added to patient education improves outcome for 2 years in adolescent patellofemoral pain: a cluster randomised trial", British journal of sports medicine, .

Rathleff, M.S., Thorborg, K., Rode, L.A., McGirr, K., Sorensen, A.S., Bogild, A. \& Bandholm, T. 2014c, "Adherence to commonly prescribed, home-based strength training exercises for the lower extremity can be objectively monitored using the Bandcizer", Journal of strength and conditioning research / National Strength \& Conditioning Association, .

Reo, J.A. \& Mercer, V.S. 2004, "Effects of live, videotaped, or written instruction on learning an upper-extremity exercise program", Physical Therapy, vol. 84, no. 7, pp. 622-633.

Salo, P.K., Hakkinen, A.H., Kautiainen, H. \& Ylinen, J.J. 2010, "Effect of neck strength training on health-related quality of life in females with chronic neck pain: a 
514

randomized controlled 1-year follow-up study", Health and quality of life outcomes, vol. 8, pp. 48-7525-8-48.

Skovdal Rathleff, M., Thorborg, K. \& Bandholm, T. 2013, "Concentric and eccentric time-under-tension during strengthening exercises: validity and reliability of stretchsensor recordings from an elastic exercise-band", PloS one, vol. 8, no. 6, pp. e68172.

Thomas, K.S., Miller, P., Doherty, M., Muir, K.R., Jones, A.C. \& O'Reilly, S.C. 2005, "Cost effectiveness of a two-year home exercise program for the treatment of knee pain", Arthritis and Rheumatism, vol. 53, no. 3, pp. 388-394.

Toigo, M. \& Boutellier, U. 2006, "New fundamental resistance exercise determinants of molecular and cellular muscle adaptations", European journal of applied physiology, vol. 97 , no. 6 , pp. 643-663.

Tran, Q.T. \& Docherty, D. 2006, "Dynamic training volume: a construct of both time under tension and volume load", Journal of sports science \& medicine, vol. 5, no. 4, pp. 707-713.

Tran, Q.T., Docherty, D. \& Behm, D. 2006, "The effects of varying time under tension and volume load on acute neuromuscular responses", European journal of applied physiology, vol. 98, no. 4, pp. 402-410.

Walther, M., Werner, A., Stahlschmidt, T., Woelfel, R. \& Gohlke, F. 2004, "The subacromial impingement syndrome of the shoulder treated by conventional physiotherapy, self-training, and a shoulder brace: results of a prospective, randomized study", Journal of shoulder and elbow surgery / American Shoulder and Elbow Surgeons .... [et al.], vol. 13, no. 4, pp. 417-423.

WMA Declaration of Helsinki 2013, , Ethical Principles for Medical Research Involving Human Subjects. Available: http://www.wma.net/en/30publications/10policies/b3/ [2014, June 4]. 
542 Figure and table legends:

543

544 Table 1: Table 1: Exercise form at follow-up

545 Figure 1: Shoulder abduction with the stretch-sensor attached to the elastic band.

546 Figure 2: Total time-under-tension at baseline and follow-up.

547

548

549

550

551

552

553

554

555 


\section{1}

Figure 1

Shoulder abduction with the stretch-sensor attached to the elastic band. 
PeerJ Reviewing Manuscript

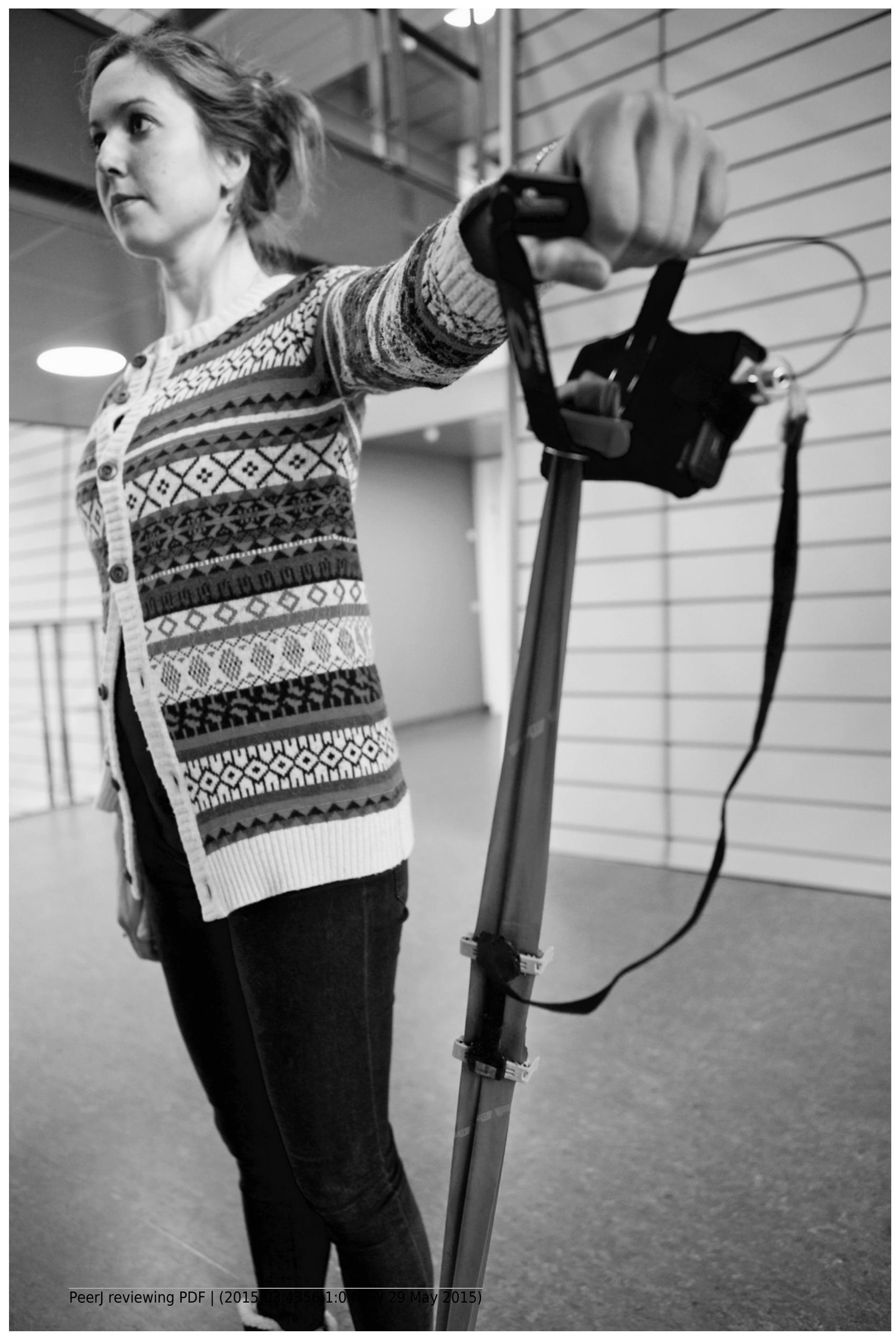




\section{Figure 2 (on next page)}

Figure 2

Total time-under-tension at baseline and follow-up. 


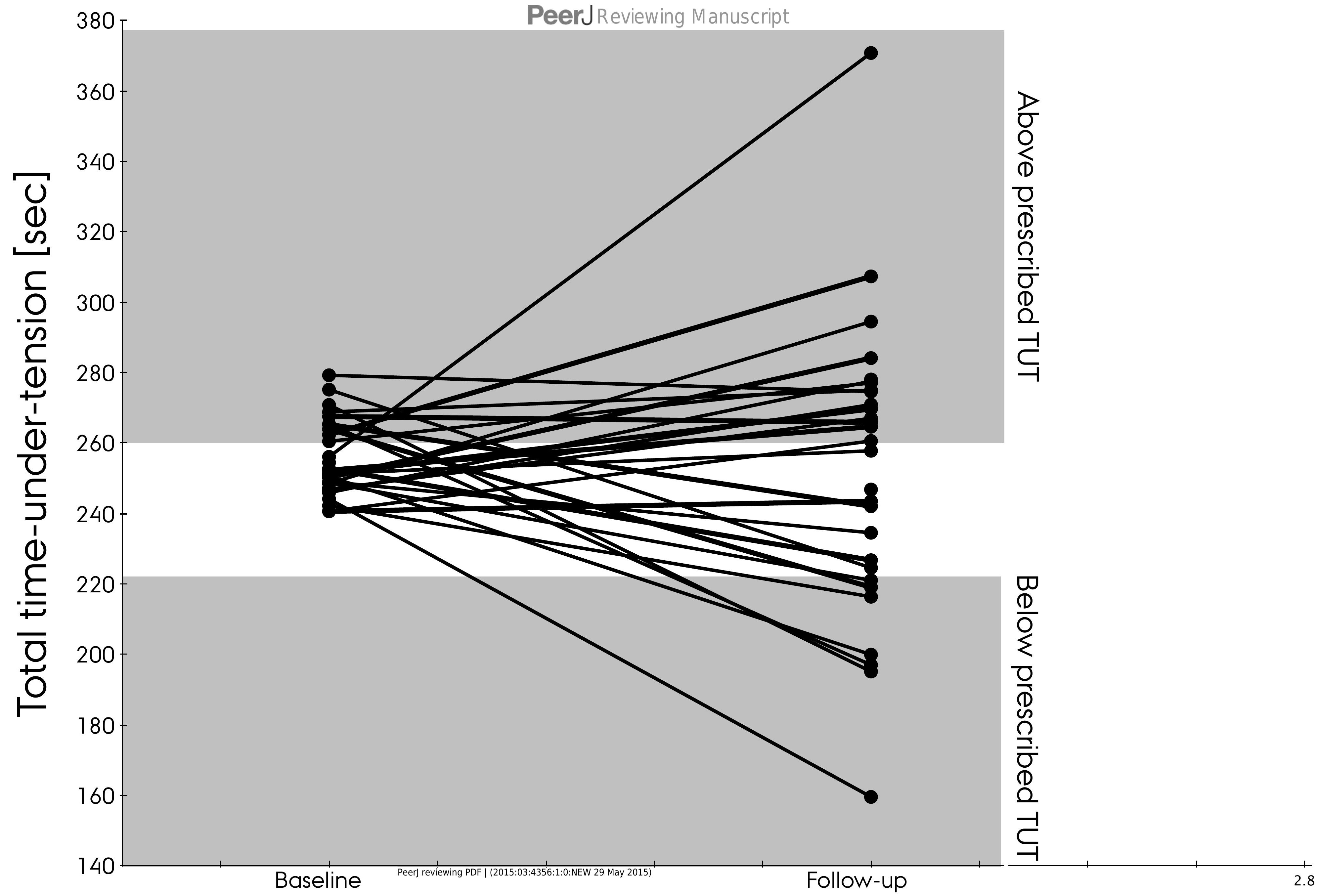




\section{Table 1 (on next page)}

Table 1

Exercise form at follow-up. 
2 Table 1: Exercise form at follow-up

\begin{tabular}{|l|l|}
\hline Exercise parameters & Number performing the exercise as instructed \\
\hline Hip width distance between the feet & $26 / 29$ \\
\hline $0-90$ degree shoulder abduction & $24 / 29$ \\
\hline 30 degree horizontal flexion & $25 / 29$ \\
\hline Palm facing the floor & $28 / 29$ \\
\hline Slight flexion of the elbow & $20 / 29$ \\
\hline $\begin{array}{l}\text { Tension in the elastic exercise band at 0 } \\
\text { degrees shoulder abduction }\end{array}$ & $28 / 29$ \\
\hline Alignment though the standing position & $29 / 29$ \\
\hline
\end{tabular}

3

4 